\title{
A Novel Approach to use Nano-Sensor in WSN Applications
}

\author{
Neeraj Kumar \\ Department of IT \\ IET, Alwar \\ Rajasthan, India
}

\author{
Amit Kumar \\ Department of IT \\ IET, Alwar \\ Rajasthan, India
}

\author{
Deepak Chaudhry \\ Department of IT \\ IET, Alwar \\ Rajasthan, India
}

\begin{abstract}
A particle that is able to sense a physical or environmental property at the nanoscale known as a NanoSensor. So it is assumed to be able to sense the properties of environmental conditions at very small and gaseous level. A nanosensor has unique properties of nanomaterials and nanoparticles to detect different events in the nanoscale. A nanosensor now assumed to be useful for the communication when it has Sensing unit, Actuation unit, Power unit, Processing unit, Storage unit, and Communication unit. In this paper we provide a framework to its use for the Mote in the wireless Sensor Networks. In this type of networks the NSU has a lot of importance that has a nanosensing unit. Inside NSU, we are suggesting to use the $\mathrm{ZnO}$ based memory due to its reliable and repeated switching of the resistance. Thus, after the combination of these two type of sensors, the network has a strong sensing capability at the nanoscale and communicating capability through WSN.
\end{abstract}

\section{General Terms}

WNSN, NanoSensor, CNT, GNR, WSN.

\section{Keywords}

CSU, ZnO, NSM,Nanosensing,,nanoactuator.

\section{INTRODUCTION}

For the communication purpose, a nano sensor is assumed with units for Sensing, Actuation, Power, Processing, Storage, and Communication.

The second part of this paper gives an introduction about above units where the sensing unit provides knowledge about the different type of Nanosensors, Actuation unit provides knowledge about different type of nanoactuators, Power unit provides the mechanism for the power consumption, Processing Unit provides the knowledge about different type of nanoprocessor including the transistors, Storage unit provides a logic for storage at the nano scale and the communication units. We have introduced to provide a brief knowledge about the technology for communication at nano scale. In the third part we are introducing the knowledge about the Wireless Sensor Node (Mote) and the Working of the Mote. In the fourth Section we are showing a logic for the use of nano sensor in Wireless Sensor network. The next sections of paper provide the assumed effects of the combination of nano sensor and Mote.

This paper provides a brief knowledge about the nanosensor, an important part of nano technology and the brief idea about its use in wireless sensor networks.

\section{NANOSENSOR}

A nanosensor has different units as per Ian et al [3] these are included here as:

Sensing unit: Based on the nature of the measured magnitude, nanosensors can be classified as- i) Physical nanosensors: they works on the properties of nanotube and nanoribbon and these are used to measure magnitudes such as mass, pressure, force, or displacement. Example of these is pressure nanosensors [21], force nanosensors [22] or displacement nanosensors [23]. ii) Chemical nanosensors: Mostly working of chemical nanosensors is based on the changes of electronic properties of Carbon Nano Tubes (CNTs) and Graphene Nano Ribbon (GNRs) change [4]. iii) Biological nanosensors: A biological nanosensor is composed of (i) a biological recognition system or bioreceptor, (ii) a transduction mechanism, e.g., an electrochemical detector, an optical transducer, or an amperometric, voltaic or magnetic detector [27]. These are used to monitor biomolecular processes such as antibody/antigen interactions, DNA interactions, enzymatic interactions or cellular communication processes. Based on this principle, nanosensors able to detect lung cancer, asthma attacks, different common virus such as the influenza virus, or the parasite responsible for malaria, have been already successfully manufactured $[5,1]$.

Actuation unit:

An actuation unit will allow nanosensors to interact with their close environment. Nanoactuators can be classified as:

Physical nanoactuators: In the way that a physical deformation of a nanotube creates a change in the electrical properties of the nanomaterial, an electrical current or electromagnetic wave can bend a nanotube [12]. These nanoactuation units will be mounted in the nanosensor device.

Chemical and biological nanoactuator: are based on the interaction between nanomaterials and nanoparticles, electromagnetic fields and heat.

Power unit:

Nanomaterials can be used to manufacture nanobatteries with high power density, reasonable lifetime and contained charge/discharge rates. i.e lithium nanobatteries. In order to overcome the limitations of nanobatteries, the concept of self-powered nano-devices has been recently introduced in $[13,6]$. The working principle of these devices is based on the conversion of the following types of energy into electrical energy: Mechanical energy, Vibrational energy, Hydraulic energy.

Processing unit:

In processing unit, main component is Nanoscale processor which is made by tinier FET Transistor and Nanomaterials such as CNT and GNR where Graphene-based transistors are not only smaller, but predictably faster.

Storage unit:

More recently, IBM Corp. has given the concept of magnetic atomic memories [14]. In a magnetic memory, single magnetic atoms are placed over a surface by means of magnetic forces [15]. Each atom can be used to store a bit [13]. 


\section{Communication unit:}

Electromagnetic communication among nanosensors will be enabled by the development of nano-antennas and the corresponding electromagnetic transceiver.

Nano-antennas: Reducing the antenna of a classical sensor device down to a few hundreds of nanometers would require the use of extremely high operating frequencies, compromising the feasibility of electromagnetic wireless communication among nanosensor devices. A few initial antenna designs based on grapheme have been already proposed. In [24], the mathematical framework for the analysis of CNTs as potential dipole antennas was developed. In [25], more emphasis was given to the numerical performance analysis of these antennas when compared to classical dipoles. In addition, several ongoing projects are conducting research on optical nano-antennas.

EM nano-transceivers: The EM transceiver of a nanosensor device will embed the necessary circuitry to perform baseband processing, frequency conversion, filtering and power amplification, of the signals that have to be transmitted or that have been received from the free-space through the nano-antenna. Taking into account that the envisioned nano-antennas will resonate at frequencies in the terahertz band, RF FET transistors able to operate at these very high frequencies are necessary. IBM Corp. has recently announced the first RF transistor made with graphene which is able to switch at $100 \mathrm{GHz}$ [2]. In [7], graphene-based frequency multipliers are implemented with grapheme.

A network of nanosensors will be able to cover larger areas and perform additional in-network processing. For the time being, it is still not clear how these nanosensor devices will communicate. We envision two main alternatives for communication in the nanoscale, namely, molecular communication and nano-electromagnetic communication: Molecular communication: Molecular transceivers will be easy to integrate in nano-devices due to their size and domain of operation. These transceivers are able to react to specific molecules and to release others as a response to an internal command or after performing some type of processing.

Nano-electromagnetic communication: this is defined as the transmission and reception of electromagnetic radiation from components based on novel nanomaterials [8].

\section{USE OF WNSN}

Biomedical applications: Biological nanosensors provide an interface between biological phenomena and electronic nano devices. Health monitoring systems: sodium, glucose and other ions in blood [19], cholesterol [26], cancer biomarkers [10] or the presence of different infectious agents [1] can be monitored by means of nanosensors. i.e. tattoo-like sensors [19].

Environmental applications: Plants monitoring systems such as trees, herbs, or bushes, release several chemical composites to the air in order to attract the natural predators of the insects which are attacking them, or to regulate their blooming among different plantations, amongst others $[16,17,20]$. Chemical nanosensors can be used to detect the chemical compounds that are being released and exchanged between plants.

Industrial and consumer goods applications: Physical nanosensors and nanoactuator can be used to enhance remote controls of complex machinery, amongst others. Similarly to the concept of distributed touch surfaces, a wireless network of nanosensors and nanoactuators could be used to enhance a peripheral such as a keyboard in cell phones or ultra-portable laptops. While a wired option may be possible, the potential of unwired nanosensors and nanoactuators is clearly higher $[9, \mathrm{i}]$. the interconnection of nanosensor and nanoactuation devices with existing communication networks and ultimately Internet enables new interesting applications that will impact also in the way we work. For example, in an interconnected office, every single element can be provided of a nanosensor device which allows them to be permanently connected to the Internet. As a result, a user can keep track of all its professional and personal belongings in an effortless fashion.

Military and defense applications: Chemical and biological nanosensors can be used to detect harmful chemicals and biological weapons in a distributed manner. However, taking into account that these sensors need direct contact with the molecules, having a network with a very large number of nanosensor nodes is necessary. Damage detection systems: physical nanosensors can be used to detect very small cracks in textiles, civil structures, vehicles and even rockets.

The interconnection of nanosensor and nanoactuator devices, existing wireless communication networks and ultimately the Internet, requires the development of new networking architectures like nanorouters and gateway. Nanorouters: these nano-devices have comparatively larger computational resources than nano-nodes and are suitable for aggregating information coming from limited nanosensor devices. nano-routers can also control the behavior of nanonodes by exchanging very simple control commands (on/off, sleep, read value, etc.). Gateway: this device will enable the remote control of the nanosensor network over the Internet. For example, in a intrabody network scenario, an advanced cellphone can forward the information it receives from a nano-micro interface in our wrist to our healthcare provider.

\section{WSN}

A wireless sensor network (WSN) is a network made by mote which has sensing, processing, transceiving power unit. This type of network is mainly useful for the military applications, and important monitoring such as health, environmental, bridge etc. Till date in the mote sensing unit using composite materials consisting of a polymer or hydrogel matrix loaded with agents specific for the detection of $\mathrm{Cl} 2$ such as NaI[11]. WSN is a self-configured, densely deployed battery operated network that is able to communicate between each other. A WSN is an ad-hoc type of network that has the features of Mobile Ad-hoc Network.

\section{PROPOSED WORK}

The idea behind the proposal is the combination of wireless nanosensor network and the wireless sensor network including the sensing unit with the nanosensors for the extra functionality of nanosensing. In the nanosensing unit the nanoscale data is taken by sensing material and passes it to the nano actuator within the actuation unit. After the processing of data it is fetched to communication unit which sent it to processing unit of sensor, here note that all these unit are battery operated, so nanobattery is also a important factor of this unit [3]. The figure 1 proposing the combination of nanosensor and the sensor where abbreviation SU presents the sensing unit, PU presents the processing unit, TU presents the Transceiver unit and NSU presents the Nanosensing Unit. In Combine Sensing Unit(CSU), we take both SU and NSU. If there is only sensing at nano scale, then only Nano Sensing Uunit communicate with the processing unit, otherwise Sensing unit communicate with the processing unit.After receiving the message from Combine Sensing unit (CSU), processing unit process the data and in case of application based network transceiver unit can receive the instruction from user or other mote and transfer it to sensing unit or nano 
sensing unit via processing unit. Processing unit also has the control over the NSM.

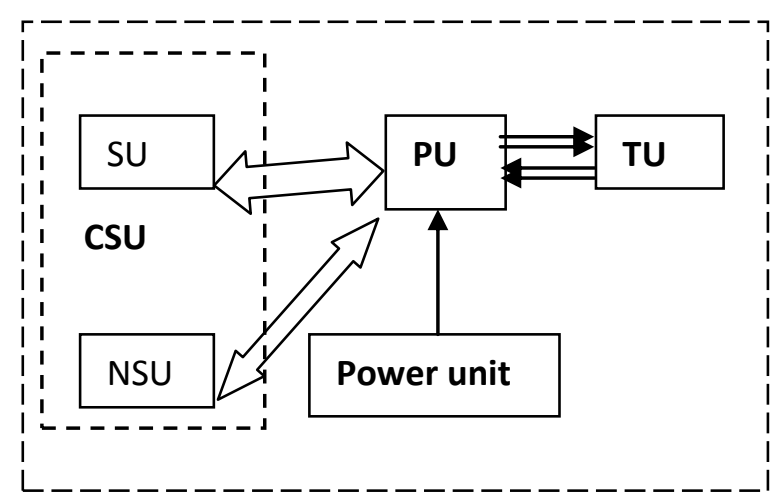

Figure1: Sensor Unit with the NSM.
PU: Processing Unit
TU: Transceiver unit
CSU: Combined Sensing Unit
SU: Sensing Unit
NSU: Nano Sensing Unit
NSM:Nano Sensing Mote

Here the Combined Sensing unit has both sensing units for molecule level sensing and for environmental sensing of a wireless sensor name as NSU and SU respectively, these two units are able to communicate between each other or separately with the processing unit when needed. Inside $\mathrm{NSU}$ we may use the $\mathrm{ZnO}$ (thinfilm, Nanobased) according to Kukreja et al[28] memory unit because $\mathrm{ZnO}$ has the large Excitation energy $(60 \mathrm{MeV})$, hard material and high temperature operable. Reliable and repeated switching of the resistance of $\mathrm{ZnO}$ thin film was obtained between two well defined states of high and low resistance with a narrow dispersion and small switching voltage.

The advantage of RRAM system (RRAM based memory unit in processing unit of NSU) differs from other competing modes are the low operating voltage and power consumption, fast switching rate, smaller and simpler bit cell structure and higher packing density. In this proposed work nanosensing unit is directly connected to sensing unit and to the processing unit NSU sense at Nanoscale and may pass the data directly to PU according to requirement.

\section{CONCLUSION}

This paper provides a brief knowledge about the nanosensor, an important part of nano technology and the brief idea about its use in wireless sensor networks. Their uses mainly include various medicinal purposes and as gateways to building other nanoproducts, such as computer chips that work at the nanoscale. In our proposed work processing unit works according to both traditional sensing unit and Nanosensing unit so it provides better sensing because it has a capability to sense at nano level.

\section{ACKNOWLEDGMENTS}

I am very thankful to my respected faculty members Prof. R.C. Tripathi (Dean, R\&D), Asso.Prof. Shekhar Verma, Asso.Prof. Shirshu Verma, Asstt.Prof. Vijay Chaurasiya, Asstt.Prof.Rajat Singh and Director of our honorable Institution Prof. M.D.Tiwari, IIITA Allahabad, and special thanks to Prof. Sudarshan Tiwari, MNNIT Allahabad and Govt. of India who provided me a lot of knowledge motivated to the research work in my school days. And also thankful to IET, alwar to provide us facility for study \& work.

\section{REFERENCES}

[1]. P. Tallury, A. Malhotra, L.M. Byrne, S.Santra, Nano bioimaging and sensing of infectious diseases, Advanced Drug Delivery Reviews 62(4_5) (2010) 424_437.

[2]. Y.M. Lin, C. Dimitrakopoulos, K.A. Jenkin , I.B. Farmer, H.Y. Chiu, A. Grill, P. Avouris, $100 \quad \mathrm{GHz}$ transistors from wafer-scale epitaxial graphene, Science 327 (5966) (2010) 662

[3]. I.F. Akyildiz, Josep Miquel Jornet, "Electromagnetic wireless nanosensor networks", Nano Communication Networks 1 (2010) 3_19.

[4]. P. Bondavalli, P. Legagneux, D. Pribat, Carbon nano tubes based transistors as gas sensors: state of the art and critical review, Sensors and Actuators B: Chemical 140 (1) (2009) 304_318.

[5] HY. Yeh, M.V. Yates, W. Chen, A. Mulchandani, Realtime molecular methods to detect infectious viruses, Seminars in Cell\& Developmental Biology 20 (1) (2009) 49_54. (A special edition on biosensors and development of pigment cells and pigment patterns).

[6]. R. Yang, Y. Qin, C. Li, G. Zhu, Z.L. Wang, Converting biomechanical energy into electricity by a muscle movement-driven nanogenerator, American Chemical Society 9 (3)(2009) 1201_1205.

[7]. H. Wang, D. Nezich, J. Kong, T. Palacios, Graphene frequency multipliers, IEEE Electron Device Letters 30 (5) (2009) 547_549.

[8]. C.Rutherglen, P.Burke, Nanoelectromagnetics: circuit and electromagnetic properties of carbon nano tubes, Small 5 (8) (2009) 884_906.

[9]. T. Hoshi, M. Takahashi, K. Nakatsuma, H. Shinoda, Touchable holography, in: SIGGRAPH, ACM, New York (NY, USA), 2009.

[10] I.E. Tothill, Biosensors for cancer markers diagnosis, Seminars in Cell \& Developmental Biology 20 (1) (2009) 55_62.

[11]. Timothy Porter, Tim Vail, Amanda Wooley, Richard Venedam, "Sensing Materials for the Detection of Chlorine Gas in Embedded Piezoresistive MicrocantileverSensors", 1190 NN1135, 10.1557/ PROC-1190-NN11-35(2009), Material research society.

[12]. C. Li, E.T. Thostenson, T.-W. Chou, Sensors and actuators based on carbon nano tubes and their composites: a review, Composites Science and Technology 68(6) (2008) 1227_1249.

[13]. Z.L.Wang, towards self-powered nano systems: from nanogenerators to nanopiezotronics, AdvancedFunctional Materials $18 \quad$ (22) (2008) 35533567.

[14]. S.S.P. Parkin,M. Hayashi, L. Thomas, Magnetic domain-wall racetrack memory, Science 32 (5873) (2008) 190_194.

[15]. M. Ternes, C.P. Lutz, C.F. Hirjibehedin, F.J. Giessibl, A.J.Heinrich, The force needed to move an atom on a surface, Science 319 (5866)(2008) 1066_1069. 
[16]. M. Heil, J. Ton, Long-distance signalling in plant defense, Trends in Plant Science 13(6)(2008)264_272.

[17] C.M. Pieterse, M. Dicke, Plant interactions with microbes and insects: from molecular mechanisms to ecology, Trends in Plant Science 12 (12) (2007) 564_569.

[18]. C.F. Hirjibehedin, C.-Y. Lin, A.F. Otte, M. Ternes, C.P. Lutz, B.A. Jones, A.J. Heinrich, Large magnetic anisotropy of a single atomic spin embedded in a surface molecular network, Science 317 (5842)2007) 1199_1203.

[19]. J.M. Dubach, D.I. Harjes, H.A. Clark, Fluorescent ionselective nano sensors for intracellular analysis with improved lifetime and size, Nano Letters 7 (6) (2007) 1827_2831.

[20] M. Heil, J.C. Silva Bueno, Within-plant signaling by volatiles leads to induction and priming of an indirect plant defense in nature, Proceedings of the National Academy of Sciences 104 (13) (2007)5467_5472.

[21]. C.Stampfer, T.Helbling, D.Obergfell, B.Schoberle, M.K. Tripp, A.Jungen, S. Roth, V.M. Bright, C. Hierold, Fabrication of single-walled CNT based pressure sensors, Nano Letters 6 (2) (2006)233_237.

[22]. C. Stampfer, A. Jungen, C. Hierold, Fabrication of discrete nanoscaled force sensors based on singlewalled carbon nanotubes IEEE Sensors Journal 6(3) (2006) 613_617.

[23].C.Stampfer, A.Jungen, R. Linder man, D. Obergfell, C.H.S.Roth, Nano-electromechanical displacement sensing based on single walled CNT, Nano Letters 7 (7) (2006) 1449_1453.

[24]. P. Burke, S. Li, Z. Yu, Quantitative theory of nano wire and nano tube antenna performance , IEEE Transactions on Nanotechnology 5 (2006) 314_334.

[25].G.W. Hanson, Fundamental transmitting properties of CNT antennas, IEEE Transactions on Antennas and Propagation 53(11) (2005) 3426_3435.

[26]. J. Li, T. Peng, Y. Peng, A cholesterol biosensor based on entrapment of cholesterol oxidase in a silica sol gel matrix at a Prussian blue modified electrode, Electro analysis 15 (12) (2003) 1031_1037.
[27].T. V. Dinh, B.M. Cullum, D.L. Stokes, Nanosensors and biochips: frontiers in bimolecular diagnostics, Sensors and Actuators B: Chemical 74 (1_3) (2001) 2_11.

[28]. N.Kukreja, A.K.Das and P.Mishra , "Studies on Non-Volatile Resistance Memory switching in $\mathrm{ZnO}$ thin film" , Indian academy of sciences, 247-253,vol. 32.

\section{AUTHORS:}

Mr. Neeraj Kumar is working as an Asstt. Prof. At IET alwar, Raj. India, has an excellent technical carrier since 2001 after completion of Diploma in Technology from Govt. Polytechnic, Budaun, India.

He received B.Tech degree in CSE from UPTU, and awarded M.Tech degree from India's most prestigious Institution, Indian Institute of Information Technology, Allahabad in IT with the specialization in wireless communication \& computing in 2005, 2010 respectively. Neeraj has more than half of dozen research papers on his research area MANET and Wireless Sensor Networks in International Journals, conferences and National Conferences. He is a organizing member of different workshop of WSN and MANET.

This is Amit Kumar working as an Asstt. Prof. at IET alwar, Raj. India, has an long academic carrier since 2000 after completion of Master in Technology (CSE).He has published various research papers on computer architecture in different international journals and conferences. He is pursuing Ph.D in computer architecture (Semiconductor memory).

Mr. Deepak Chaudhary has completed his M.Tech (SE) in 2010 from Thapar University,Patiala,(Punjab) after completion of M.Sc in. Now, he is an Asstt.Prof. in IET, Alwar, Raj., India, and a student member of IEEE. 